\title{
Chapter 4 \\ Morphological Variation in Birds: \\ Plasticity, Adaptation, and Speciation
}

\author{
Till Töpfer
}

\begin{abstract}
The huge diversity of phenotypes and associated geographic patterns has made birds prime examples for studies in speciation. For this purpose, morphological approaches were first choice to assess the degree of relatedness between species and their intraspecific variation for centuries, until molecular genetic studies seriously challenged traditional morphology-based conclusions. However, the current development of multivariate statistics and the ease to blend morphological, phylogenetic, and ecological insight has gradually led to a reconsideration of morphology as a valuable tool for ornithological research. This chapter reviews the most important aspects of morphological variation in birds, how its plasticity can be assessed and to which extent phenotypic variation can be incorporated into a broader evolutionary framework that explains modifications of the avian body in the light of speciation processes.
\end{abstract}

Keywords Avian morphology · Phenotypic plasticity · Trait evolution · Geographic variation · Eco-morphology

\subsection{General Aspects of Phenotypic Variation in Birds}

Studying speciation means to analyze the variation of characters, no matter if on molecular or organismic level. In birds, a great deal of such variation concerns phenotypic traits, making morphological assessments an important tool to collect evidence both for identification and classification. Identification of birds is intuitively associated with their external appearance. In contrast to many other animal groups, birds are commonly perceived as more or less readily distinguishable on grounds of size, shape, coloration, song, and other behavior. Being abundant and popular for centuries, birds remain to be subjects of both popular pastime and scientific

\footnotetext{
T. Töpfer (四)

Zoological Research Museum Alexander Koenig, Centre for Taxonomy and Evolutionary

Research, Section Ornithology, Bonn, Germany

e-mail: t.toepfer@leibniz-zfmk.de 
occupation (Birkhead et al. 2014). At the core of this lies the study of differentiation of bird populations using comparative approaches. Although the distinction of certain species might differ if different conceptual principles are applied, phenotypic characters persist as relevant criteria for the delimitation of bird species.

In contrast to the common and oftentimes inevitable use of identification keys, as in most invertebrates, there is no comprehensive identification key of birds to date. A major reason is that in no other vertebrate group the legacy of ancient pictorial accounts remains as strongly as in birds - the huge number of contemporary identification guides, regardless of being in printed or digital form, speaks for itself. It is a compelling thought that such guides effectively combine depictions and descriptions in a time-honored way that have their roots in the narrative accounts of the first popular books of natural history. Even though modern analytical methods, spearheaded by molecular genetics, have shaken our understanding of species boundaries in birds, we still strongly rely on phenotypic assessment in species delimitation and classification. So, why are the external characteristics of birds so decisive that they still form a touchstone of species identity?

First and foremost, it is the enormous range of morphological variation in birds despite their relatively similar body plan. Even on lower taxonomic level, with resembling appearances of closely related taxa as a rule, the diversity of structural and coloration patterns is striking. Since many of these differentiations can be interpreted as functional adaptations to a certain lifestyle and/or to environmental conditions, they tell a lot about ecological causes of phenotypic differentiation. This, in turn, fosters our understanding of the evolutionary constraints that may eventually lead to speciation. Considering the current development of multidisciplinary approaches in evolutionary research, it is worthwhile to have a detailed look at the different aspects of morphological variation revolving around the problem of speciation in birds.

\subsection{The Historical Role of Morphological Criteria for Species Delimitation}

Morphology in its broadest sense, i.e., comprising all manifestations of structure and form, has been most influential for the classification of biological beings over centuries. As a basic ordering principle, morphology was applied to organize a seemingly chaotic variety of life forms on higher ("macrotaxonomic") levels first (Mayr 1982). When the scientific interest focused on lower, species-level ("microtaxonomic") classification, the desire grew to also reflect phylogenetic relationships in taxonomy. In any case, morphological approaches remained unrivalled to assess the degree of relatedness between species and their intraspecific variation until the 1990s, when techniques of molecular genetics, particularly DNA sequencing, became broadly available. This held true for ornithology as well. Except for bioacoustics (Baptista and Kroodsma 2001), that had a substantial influence on testing 
species boundaries experimentally (at least in vocally active birds), there was hitherto no other noteworthy method to gather relevant evidence (Box 4.1).

The advent of molecular methods and the rapidly growing computational power enabled a previously nonexistent phylogenetic interpretation of extensive data sets and stimulated an extremely fruitful discourse about speciation in birds by challenging various traditional morphology-based classifications. During the subsequent phase of rapid upheavals in avian systematics that also had a massive impact on the huge ornithological community beyond academic circles, the importance of morphology for species identification and classification has been widely challenged. This was actually a renewed flaring-up of the old discussion to which extent morphology actually reflects phylogeny. However, having meanwhile entered the era of genomics that produces fascinating deeper insight into avian evolution, it turned out that interdisciplinary approaches are the means of choice to further elucidate the riddles of speciation in birds. The development of multivariate statistics and the ease to blend morphological, phylogenetic, and ecological parameters has gradually led to a reconsideration of morphology as a valuable tool for ornithological research.

\section{Box 4.1 The Importance of Ornithological Collections}

Bird collections, either in museums or in private hands, have been the cradle of comparative studies on character variation from the beginning of scientific ornithology. Providing a well-documented and geographically widespread material basis for analyses of differentiation patterns, they have fueled the debates about species delimitation and conceptual issues of speciation and classification (Bruce 2003). Having previously been considered sources for morphological and geographical information in the first place, the use of ornithological collections has experienced a major extension when bird specimens have been shown to represent invaluable resources for molecular genetic research as well. As a consequence, many bird collections store additional samples for molecular genetic analyses together with classic skin or skeleton specimens nowadays. Considering the growing interest in integrative research approaches (e.g., Alström et al. 2016), combining studies of variation on very different biological levels, ornithological collections will continuously contribute to scientific progress by further archiving and documenting vouchers of contemporary avian diversity (Webster 2017).

\subsection{Phenotypic Variation and Plasticity of Characters}

The fascinating diversity of their phenotypes and associated geographic patterns has made birds prime examples for studies in speciation. However, like in other groups of animals, there is not always an immediate connection between external appearance and species identity. In order to correctly distinguish between interspecific differences and intraspecific variation, it is inevitable to scrutinize the extent of 
geographic and individual variability within and between potential species (Mayr 1969). As the limits of such variation may shift temporarily as a result of adaptive processes (e.g., Grant and Grant 2014), character plasticity has to be considered likewise. Of course, this does not only concern morphological traits. A plethora of molecular studies has shown that the variation of genetic characters (i.e., DNA sequence polymorphisms) is a powerful tool to define groups of organisms even on a very fine scale. This is especially valuable when combining morphological and molecular evidence. In the following, however, only the variation of external characters will be in focus.

Geographic Variation Geographic variation refers to the differentiation between different populations (Mayr 1969). Obviously, geographic variation covers all aspects of phenotypic differentiation on various geographic scales (Cicero and Remsen 2007). There is no rule as to how large geographic distances have to be until morphological differentiation sets in, but evidently at least some (historical) amount of geographic isolation (vicariance) appears necessary (Price 2008). Consequently, geographic variation often is not only pronounced in birds with a wide distribution but also in those that inhabit ecologically fragmented ranges on smaller geographic scales. Traditionally, populations of these polytypic species are grouped as subspecies based on the particular composition of morphological traits at a given locality (Winker and Haig 2010).

Geographic differentiation can either be continuous (clinal) or discontinuous. While the latter is commonly found in patterns of insular distributions or wide geographic range separations (disjunct distribution), the former frequently occurs in more or less continuous continental ranges. Clinal variation, however, can well be incongruent between different phenotypic traits indeed. As a result of such incongruently directed character changes, each bird population at any intersection of different clines represents a certain morphological entity with a particular trait combination.

As with other forms of morphological variability, geographic variation is not restricted to particular traits and can basically affect any aspect of a bird's physique. What makes it even more interesting in evolutionary respect is the often-found correlation between geographic variation and ecogeographic rules (e.g., Sun et al. 2017), indicating the selective effect of environmental conditions on the avian body. Moreover, eco-morphological insight, such as the connection between wing structure and flight performance or migratory distance, may be likewise reflected in inter- and intraspecific geographic variation (Winkler and Leisler 2005).

Individual Variation By definition, individual variation comprises all phenotypic manifestations at the same locality (Mayr 1969). Such local variation, however, does not necessarily need to be less pronounced than geographic variation. There are different forms of individual variation and all of these forms may be represented by interindividually variable characters again. Moreover, the forms of individual variation are not exclusive as they might occur even in the very same individual at different ages or seasons. The most frequent forms of individual variation in birds are sexual, age-dependent, and seasonal variation, complemented by local eco-morphological differentiation within populations. 
Sexual Variation Sexual variation includes all aspects of phenotypic differentiation between the two sexes. This is of course most obvious in those birds that exhibit a pronounced sexual dimorphism, be it in size, coloration (patterns), or in both. Sexual dimorphism does not need to be constant throughout the year, as more conspicuous breeding plumages might differ more strongly between the sexes than non-breeding plumages, indicating the overlap with seasonal variation. In addition, sexual dimorphism may not only be due to sexual selection alone but might also have an ecological component. In many raptors (Falconidae, Accipiter), there is a substantial size difference between the two sexes with the larger females apparently exploiting a different food spectrum than the smaller males. Within females and males, again, there is variation in the respective characters that defines the extent of sex-specific characteristics. Other special cases are those birds in which the external appearance of one sex is strongly correlated with specific aspects of the mate choice process and where strong sexual selection leads to extreme phenotypic diversity within one sex. The prime example is the Ruff Philomachus pugnax, whose males are individually distinct in plumage patterns and coloration. But there is not only the males' extreme plumage variability (that can be subdivided into main character groups) but also the existence of female-mimicking males that also form an intermediate size group in wing length between "proper" males and females (Jukema and Piersma 2006).

Age-dependent Variation Regarding age-dependent variation, a broad range of character differences have to be considered. As far as the feather coat is concerned, consecutive plumage generations of the same individual are involved. A precise knowledge of molt sequence is thus necessary to properly assess this kind of phenotypic variation (e.g., Jenni and Winkler 1994). It is the nature of things that during molt transitional plumage stages occur and that particularly in long-lived birds (e.g., eagles and vultures) persistent composite plumages exist for years that comprise feathers of several generations. As a rule of thumb, a distinction can be made between plumages worn before becoming reproductively active (juvenile and so-called immature plumages) and those worn by reproductively active birds (adult plumages). Nonreproductive plumages might differ in terms of dimensions, structure, and coloration and patterns in being shorter, differently shaped (particularly in the large flight feathers), appearing more fluffy and being less strikingly colored and patterned. In addition, it is commonly the case that nonreproductive plumages are very similar between sexes even if the adult plumage is clearly sexually dimorphic. Moreover, there is growing evidence that bird plumages may also be subject to senescence ("progressive greying," van Grouw 2013). As birds apparently remain reproductively active until their death, however, this stage has to be considered a representation of the adult plumage as well. But not only plumage traits are subject to age-dependent variation. Other physical features like iris, leg, or bill color as well as structural differences because of not yet full-grown characters (e.g., bill size and shape) might be concerned likewise.

Seasonal Variation Another often-found form of variation is seasonal: In the course of the year, many birds acquire two different plumages of which one usually is more conspicuous. Since in most cases such conspicuousness is explained by the demands of mate choice and breeding, the breeding plumage tends to be more prominent - at 

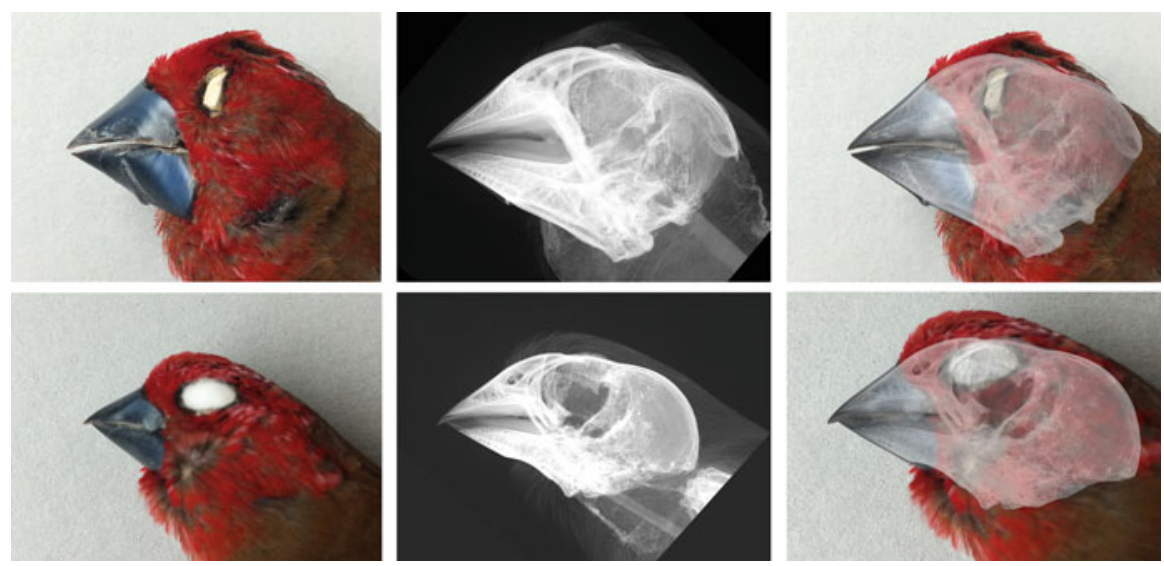

Fig. 4.1 Variation of bill dimensions in the Pyrenestes seedcrackers (specimens from the Zoological Research Museum A. Koenig). Top row, P. ostrinus; lower row, P. minor. Specimen profiles are shown in external view, in X-ray view, and as a superimposition of external and X-ray views, respectively. Such extreme intraspecific variation of an external character is also reflected in the internal anatomy as obvious from the X-ray pictures of the individual skulls

least in one sex (cf. sexual variation). In the more unobtrusively colored or patterned non-breeding plumages, possible phenotypic differences between males and females might become even less pronounced. In a number of birds, particularly among nonmigratory species of the temperate regions, seasonal variation in external characteristics is an adaptive way to cope with substantially changing environmental conditions. One of the most striking examples is the difference between the summer plumage and the winter plumage of some ptarmigan Lagopus species which permits a seasonally efficient camouflage throughout the year. Similar to the effect in age-dependent plumages, there are also transitional plumage stages consisting of feathers of two different, although recurringly molted, feather generations.

Resource Polymorphism As described above, patterns of phenotypic variation can be complex and causally overlapping. To this adds the fact that in some birds, apparently as an adaptation to quickly changing resources within habitats, there is a corresponding swift eco-morphological response between and within species, as most prominently described for Darwin's finches (Grant and Grant 2014). The so-called resource polymorphism has also been studied extensively in Pyrenestes seedcrackers (Fig. 4.1) in which the size and structure of their bills differ at the same time between individuals of a single population that have adapted to different food items (Smith 1990).

Phenotypic Plasticity The preceding paragraphs show that morphological variability concerns different manifestations of external differentiation on individual, population, or geographic level and that sometimes several forms of variation might even overlay. Collectively, they represent the phenomenon of phenotypic plasticity. Premising the idea that selection acts on the phenotype, it is important to keep in mind that character variation as such is not a result of evolutionary adaptation in the first instance rather than a mean to flexibly enhance the evolutionary success of a 
population by stochastically offering variants of morphological traits for selective processes. The phenotype resulting from natural selection of advantageous traits may then represent a particular fraction of previous generations' character variation and again might have its very own range of variation. As phenotypes do also reflect a response to local or temporal ecological conditions, which are not fixed over time themselves, a great deal of morphological plasticity in time can be likewise observed within or between populations and species (e.g., Grant and Grant 2014). Ultimately, shifts in character variation between populations can (but do not have to) lead to evolutionary distinct units that might carry the germ of new species (cf. Yousefi et al. 2017). Therefore, particularly if preserved specimens are part of studies of avian speciation, researchers have to carefully select the geographic and temporal origin of the specimens.

\subsection{Assessing Morphological Variation}

Just as diverse as morphological variation itself is the number of methods to assess it properly. As soon as ornithologists became aware of the evolutionary and ecological importance of understanding phenotypic variation that hitherto had been documented mainly descriptively with a focus on identification, the need for objective and reproducible methods grew. Through the common use of multivariate statistics, a great deal of stimulating functional, ecological, and evolutionary interpretations emerged. The technical progress of the last decades further supported the application of computer-based assessments of morphological traits. For the assessment of size and proportions, an array of two- (linear) and three-dimensional methods of measurement is currently available and gets constantly modified and extended. Combined with sophisticated statistical analyses, they allow complex descriptions of the avian body and its modifications. In a similar attempt to objectivize color assessments of plumage and bare parts, advanced methods of spectral analyses are applied, and the description of color patterns is currently being modernized by the application of pattern recognition software (Bostwick et al. 2017; Burns et al. 2017).

Linear Measurements As a classic method of assessing morphological variation, linear measurements are routinely taken in almost every scientific ornithological study. Explanations and recommendations of methods are well-documented in the literature (e.g., Baldwin et al. 1931; Svensson 1992; Eck et al. 2011). However, considering the fact that most biometric methods often rely on a set of more or less established measurements, students of morphological variation need to be aware that such standard measurements might capture only certain morphological aspects of the avian body (Box 4.2). Nonetheless, these measurements are very helpful to assess not only variation per se but will also generate data allowing the assessment of functionally relevant complexes such as the flight, walking, and feeding apparatus (Fig. 4.2). 


\section{Box 4.2 Avian Body Size}

Although seemingly trivial, the comparative assessment of a bird's body size is not entirely straightforward. As the avian body construction is generally shaped to reduce body mass, there is a massive constructional pressure on the trunk, whereas limb and neck bones as well as the large flight feathers, though often extensive in their dimensions and functionally important, may contribute considerably less to the overall body mass in many birds.

Having arisen from traditional studies of preserved specimens, a relatively small number of mostly linear standard measurements are routinely taken in ornithological research, both for live and preserved birds (Eck et al. 2011). This is due to the fact that the techniques of bird taxidermy (essentially skinning the bird and coating an artificial replacement body with that skin, e.g., Winker 2000) allow only a limited number of reliable comparative measurements, once the study skin has been made (Fig. 4.2).

As a consequence, some biologically relevant measurements cannot be taken any more (Fig. 4.2). Therefore, irretrievable morphometric data (e.g., wingspan, total body length, body mass) need be documented during the preservation process of bird specimens in order to guarantee the comparability of data with those taken from live birds. Because of the scarcity of such (statistically meaningful) data in many groups, authors have used body mass data as a proxy for body size instead. Due to the computational power of contemporary statistical analyses, however, a combination of several morphological traits, including morphometric data and body mass, appears to be the most promising avenue to come to biologically sound conclusions.
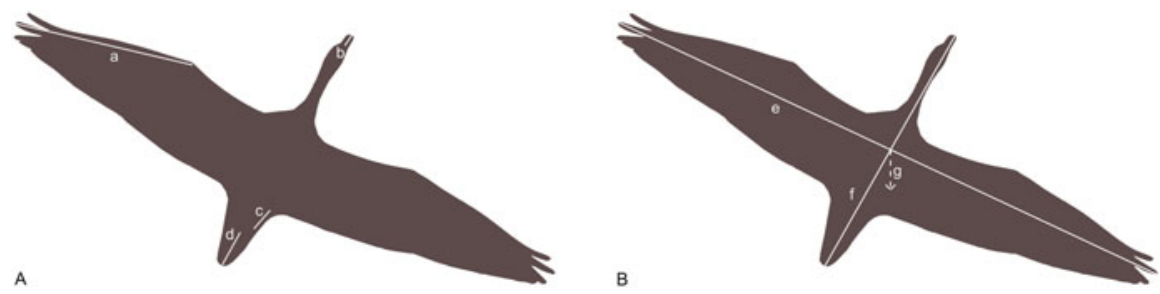

Fig. 4.2 Morphometric assessment of avian body size. (A) Classical partial measurements, usually taken from study skins; (B) biologically relevant total measurements, meaningful for flight performance analyses. While measurements under (A) can be re-collected from specimens virtually at any time, those under $(\mathbf{B})$ are irretrievable from study skins and must be taken from the fresh dead or live bird. a, wing length; $b$, bill length; $c$, tarsus length; $d$, tail length; $e$, wing span; $f$, total body length; g, body mass

Three-dimensional Measurements Three-dimensional scanning techniques that complement classic linear measurements are not only desired because of the reproducibility and precision of digital data but also because they allow more complex reconstructions of morphological shapes and proportions that are hardly accessible 
by linear measurements. Although a great deal of work is still done manually by setting digital landmarks for final calculations, it is foreseeable that these processes will become more and more automated, thus further accelerating the speed of analyses. As three-dimensional visualizations principally allow minimally invasive analyses of the inner body as well (e.g., via CT scanning), a number of new findings will additionally enlighten the connection between phenotype and internal characteristics of birds (James 2017).

Color Assessment By contrast, the assessment of plumage and soft part colors traditionally is a descriptive one. Because the individual perception of colors is often incongruent between observers, in particular regarding more subtle color differences, color charts have been developed to objectivize the assessment. There is a number of such charts available, sometimes exclusively designed to meet the requirements of biologists (e.g., Ridgway 1912), but practical problems remain as different editions of the same charts might differ because of the respective printing processes. A fine-scaled comparability of results is only guaranteed if the charts from the very same edition are compared (and if the charts were stored under similar conditions). Meanwhile, spectrophotometric analyses have complemented descriptive methods (Burns et al. 2017). By studying the reflectance spectra of color patches, the presence of pigments and light-reflecting feather microstructures can be inferred based on wavelength-specific reflection patterns that also allow a quantification of involved pigments. Another important aspect of spectrophotometry is the detection of ultraviolet components of plumage color that are not visible by humans but apparently play a role in avian signaling as birds are able to see in the UV range. Spectral color data can be incorporated in similar statistical analyses like morphometric data, making virtual reconstructions of so-called color spaces possible. However, spectral analyses have not replaced classic descriptive approaches completely yet. Not only that for some research questions it would simply be exaggerated to expend high-end technological effort, its application is still relatively laborious considering data collection and analysis. It is thus likely that in the future a well-balanced combination of both traditional and spectral color analyses will be performed instead of a complete replacement of descriptive methods.

Pattern Analyses Nonetheless, color assessment does not only comprise the precise identification of a color but also its distribution on a bird's body. Even more than color identification, pattern analysis is mostly a descriptive procedure. Considering the huge complexity of many bird plumage (or egg shell) patterns, it is obviously difficult to apply a single simple method for comparative studies. Therefore, researchers have chosen different approaches to describe the shape and distribution of color patterns depending on the focus of their study and the group of birds considered (Bostwick et al. 2017; Burns et al. 2017). The more complex these patterns are, the more often partitioned approaches are applied, concentrating on analyzing parts of the avian body first before recomposing the whole pattern information. With the aid of pattern recognition software, however, a great leap forward was made to effectively analyze and compare external patterns in birds. To 
date, most of the studies still focus on the analysis of two-dimensional data, using digital photographs of objects. Nonetheless, the most useful way to apply digital techniques to pattern recognition in ornithology is the assessment of threedimensional data in order to reflect the true distribution of patterns on the avian body properly. Considering the current speed of technical advance in the field of three-dimensional pattern recognition in general, such technology will likely become available for scientific bird research very soon.

\subsection{Disentangling Phylogenetic and Adaptive Constraints}

Probably the most arduous challenge in the study of phenotypic variation is to detect if it reflects phylogenetic or adaptive constraints. In evolutionary respect, morphological plasticity within and between bird species is a result of natural selection that might produce convergent phenotypes under similar extrinsic pressures for functional reasons. Consequently, externally resembling bird taxa do not necessarily need to be closely related (and vice versa), and morphological uniformity might disguise cryptic diversity on species level. Indeed, both polymorphism and cryptic diversification can obstruct our view on species distinction. While the former phenomenon nowadays poses a much smaller difficulty in terms of species assessment because of molecular genetic counterchecking, the latter still is less easy to detect and often only discovered if other methods have provided additional evidence. The task of disentangling phylogenetic and adaptive constraints when studying morphological variation is all the more compounded by the fact that phylogeny and adaptation are usually not independent of each other. During trait evolution, as a response to selective pressures, adaptive processes shape the morphological composition of a population which becomes subsequently fixed itself during phylogeny. It is therefore reasonable to assume that present morphological variation reflects a causally mixed result of trait evolution within an avian lineage. Apparently, there is no infinite plasticity of characters within avian lineages. Once a given set of characters is acquired within a lineage, a certain extent of modification can be observed, but rarely revolutionary reconstructions.

Regarding the question if a morphological character is a relevant feature of species distinctness or just an adaptation to similar ecological conditions, current multidimensional statistical analyses can identify potential phylogenetic effects in a morphological data set. However, credible phylogenetic hypotheses have to be available a priori which usually are based on molecular genetic data. Potentially, the emerging field of population genomics might provide other reliable data (BoteroCastro et al. 2017). However, without the support of additional information, the interpretation of morphological variability as species-delimiting criteria still lies in the eye of the beholder. 


\subsection{A Contemporary Perspective on Morphological Variation}

We have seen that studies of morphological variation are important elements of multidisciplinary studies by combining classic and modern approaches. Having become modernized themselves by sophisticated statistical analytic tools, they do not only complement other cutting-edge methodologies but add considerable new input to our contemporary understanding of bird evolution and adaptation. In combination with growing insight into phylogenetics and ecology of birds, reconstructions of trait differentiation are not limited to the study of few avian lineages but extendable to eco-morphological analyses within and between communities at any geographic dimension ("integrated eco-morphology", Leisler and Schulze-Hagen 2011). It is to be expected that such analyses will become possible even back in time provided enough comparative data (from historical specimens or fossils) can be accessed. The rapidly evolving field of (population) genomics will surely do its share here. In the long run, it would be desirable to integrate data on phenotypic variation into a broader evolutionary framework that also explains modifications of the avian body in the light of speciation processes. By all means, the study of morphological variation will remain a crucial element of ornithological studies, not only when dealing with speciation. The current technical advances allow a broad range of investigations that will deliver important insight into the evolutionary differentiation of birds way beyond than just telling species apart.

\section{References}

Alström P, Rasmussen PC, Zhao C, Xu J, Dalvi S, Cai T, Guan Y, Zhang R, Kalyakin MV, Lei F, Olsson U (2016) Integrative taxonomy of the Plain-backed Thrush (Zoothera mollissima) complex (Aves, Turdidae) reveals cryptic species, including a new species. Avian Res 7:1

Baldwin SP, Oberholser HC, Worley LG (1931) Measurements of birds. Sci Publ Cleveland Mus Nat Hist 2:1-165

Baptista LF, Kroodsma DE (2001) Avian bioacoustics. In: del Hoyo J, Elliott A, Sargatal J (eds) Handbook of the birds of the world, vol 6. Lynx Edicions, Barcelona, pp 11-52

Birkhead TR, Wimpenny J, Montgomerie B (2014) Ten thousand birds. Ornithology since Darwin. Princeton University Press, Princeton

Bostwick KS, Harvey TA, Scholes E III (2017) Leveraging diverse specimen types to integrate behavior and morphology. In: Webster MS (ed) The extended specimen. Emerging frontiers in collection-based research. CRC Press, Boca Raton, pp 75-88

Botero-Castro F, Figuet E, Tilak MK, Nabholz B, Galtier N (2017) Avian genomes revisited: hidden genes uncovered and the rates versus traits paradox in birds. Mol Biol Evol 34:3123-3131

Bruce M (2003) A brief history of classifying birds. In: del Hoyo J, Elliott A, Sargatal J (eds) Handbook of the birds of the world, vol 8. Lynx Edicions, Barcelona, pp 11-43

Burns KJ, McGraw KJ, Shultz AJ, Stoddard MC, Thomas DB (2017) Advanced methods for studying pigments and coloration using avian specimens. In: Webster MS (ed) The extended specimen. Emerging frontiers in collection-based research. CRC Press, Boca Raton, pp 23-55 
Cicero C, Remsen JV (2007) Festschrift for Ned K. Johnson: geographic variation and evolution in birds. Ornithol Monogr 63:1-114

Eck S, van den Elzen R, Fiebig J, Fiedler W, Heynen I, Nicolai B, Töpfer T, Winkler R, Woog F (2011) Measuring birds. Vögel vermessen. Wilhelmshaven, Deutsche Ornithologen-Gesellschaft

Grant PR, Grant BR (2014) 40 years of evolution. Darwin's finches on Daphne Major Island. Princeton University Press, Princeton and Oxford

James HF (2017) Getting under the skin. A call for specimen-based research on the internal anatomy of birds. In: Webster MS (ed) The extended specimen. Emerging frontiers in collection-based research. CRC Press, Boca Raton, pp 11-22

Jenni L, Winkler R (1994) Moult and ageing of European passerines. Academic, London

Jukema J, Piersma T (2006) Permanent female mimics in a lekking shorebird. Biol Lett 2:161-164

Leisler B, Schulze-Hagen K (2011) The reed warblers: diversity in a uniform bird family. KNNV Publishing, Zeist

Mayr E (1969) Principles of systematic zoology. McGraw-Hill, New York

Mayr E (1982) The growth of biological thought. Belknap Press, Cambridge, MA

Price T (2008) Speciation in birds. Roberts and Company, Greenwood Village

Ridgway R (1912) Color standards and color nomenclature, Washington, DC

Smith TB (1990) Natural selection on bill characters in the two bill morphs of the African finch Pyrenestes ostrinus. Evolution 44:832-842

Sun Y, Li M, Song G, Lei F, Li D, Wu Y (2017) The role of climate factors in geographic variation in body mass and wing length in a passerine bird. Avian Res 8:1

Svensson L (1992) Identification guide to European Passerines, 4th edn. Ugga, Stockholm

van Grouw H (2013) What colour is that bird? Br Birds 106:17-29

Webster MS (2017) The extended specimen. Emerging frontiers in collection-based research. CRC Press, Boca Raton

Winker K (2000) Obtaining, preserving, and preparing bird specimens. J Field Ornithol 71:250-297

Winker K, Haig SM (2010) Avian Subspecies. Ornithol Monogr 67:1-200

Winkler H, Leisler B (2005) To be a migrant: ecomorphological burdens and chances. In: Greenberg R, Marra PP (eds) Birds of two worlds: the ecology and evolution of migration. John Hopkins University Press, Baltimore, pp 79-86

Yousefi M, Kaboli M, Eagderi S, Mohammadi A, Rezai A, Nourani E (2017) Micro-spatial separation and associated morphological adaptations in the original case of avian character displacement. Ibis 159:883-891

Open Access This chapter is licensed under the terms of the Creative Commons Attribution 4.0 International License (http://creativecommons.org/licenses/by/4.0/), which permits use, sharing, adaptation, distribution and reproduction in any medium or format, as long as you give appropriate credit to the original author(s) and the source, provide a link to the Creative Commons license and indicate if changes were made.

The images or other third party material in this chapter are included in the chapter's Creative Commons license, unless indicated otherwise in a credit line to the material. If material is not included in the chapter's Creative Commons license and your intended use is not permitted by statutory regulation or exceeds the permitted use, you will need to obtain permission directly from the copyright holder.

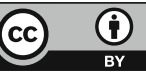

